\title{
Vehicle Recognition based on Pseudo invariant Linear Moment Features and ELM
}

\author{
Shao Yong-lin \\ CAD/CAM Institute of Central South University \\ Changsha, Hunan, China
}

\author{
Luo Yi-ping \\ CAD/CAM Institute of Central South University \\ Changsha, Hunan, China
}

\begin{abstract}
In view of the problem of slow speed and low accuracy of the vehicle recognition in advanced driver assistance systems, a vehicle recognition method based on pseudo invariant linear moment features and ELM is proposed. Target edge is extracted by the improved PCNN model, according to the characteristic of multiple target features, the pseudo invariant linear moment features are extracted, then ELM model is used to train and recognize the databases. The validity of the model is verified through experiments, compared with other algorithms, the recognition accuracy of pseudo invariant linear moment features and ELM vehicle recognition method is higher and the speed is faster, which provides a new way to identify the vehicle in real-time monitoring system of the vehicle.
\end{abstract}

\section{Keywords}

Line moment; feature extraction; target recognition

\section{INTRODUCTION}

About 125 million people died as a result of road traffic accidents every year around the world according to the World Health Organization for the year 2015 global road safety status report statistics, and the resulting medical property losses spend up to $3 \%$ of the total expenditure of medical care [1]. Therefore, with the aim to reduce the traffic accidents and improve the traffic safety has becoming an area of traffic research among the people (including the drivers, passengers and pedestrian), vehicles, road and the environment contradiction. Owing to the development of camera sensing and computing technology, image processing technology has immensely progressed.

Advanced Driver Assistance Systems (ADAS [2]) refers to the use of vehicle equipment to collect the internal and external environment data and operational analysis the risk of possible early warning to improve the safety of driving. How to make accurate, reliable and effective identification of vehicles in complex scenes is a big challenge for ADAS. Vehicle recognition and tracking based on visual image processing is a hot topic in the field of traffic engineering, and it is also an effective method to solve this problem. Different vehicle identification methods are proposed, V. D. et al. [3] provides a survey of recent works of vehicles detection, tracking and behavior understanding on road based on vision.

\section{RELATED WORK}

At present, scholars have introduced the pattern recognition to the vehicle identification based on the video image to feature description and classifier design as the core of the two classification modeling. Among them, the overall image feature description is mainly related to color, shape and texture, local image feature description involving SIFT features, SURF features, class Haar features, classifier design and application of a wide range of Adaboost, PNN, BP and
SVM, etc. Cai et al. [4] proposes a method of samples selfmarking transfer learning by PNN to reduce training time and improve the vehicle recognition ability. JIN et al. [5] introduces a algorithm of Haar-like features and Adaboost to improve the capability of ADAS. Those proposed methods can realize real-time vehicle recognition under the specific scene. However, owing to different perspective, weather, color, background and other real road scenes factors, the accuracy and reliability of vehicle recognition is not reached satisfactory degree. By analyzing and summarizing the existing methods of feature description and classifier, this paper introduces the Pulse Coupled Neural Networks (PCNN) model and the Extreme Learning Machine (ELM) algorithm, a vehicle recognition method is proposed, which we use the properties of pulse coupled neural network dynamic neurons, synchronous pulse radiation and gray scale invariant, to extract the image edge information quickly and effectively, then extract pseudo invariant line moments according to the edge information, finally use the advantages of extreme learning machine learning speed and generalization capability to classify the target. After vehicle recognition is experimented on static pictures, we can see the feasibility and validity of the model.

\section{BACKGROUND}

\subsection{PCNN Image Edge Extraction}

Based on the cat and monkey cerebral cortex neuron dynamic characteristic, Jonson and Padgett et al proposed the linking field network which became the pulse coupled neural network $(\mathrm{PCNN})$. Compared with the traditional artificial neural network, the PCNN have the advantages of rotation invariant, scale invariant, translation invariant and gray intensity invariant, which be found that it is suitable for real-time image processing including image segmentation, feature extraction, pattern recognition and communication[6-8].

Image edge is the singular point and point mutations of the gray image, which are mainly manifested in the discontinuous region of the local characteristics. In order to extract the demarcation line between the targets and background as well as the dividing line between the targets, the differential difference and gradient operation used to detect, which include Canny operator, Sobel operator, Prewit operator and Laplaican operator. The output of the PCNN process is the extraction process of edge texture and region information through the analysis of the PCNN principle and image processing $[9,10]$. However, a single ignition output can only display part of the image edge information which means a single iteration output may be missing some other edge information of original image. In order to resolve the problem the method that a parameters adaptive PCNN edge extraction is proposed, transforming the input image into gray image, then removed the target edge by erosion operation, by comparing to the corrosion image and the original image we can get multiple internal and external contour line of target characteristics. 


\subsection{Review of ELM}

The ELM we used as classifier is proposed which is a special single-hidden layer feed forward networks (SLFNs). The essence of ELM is that the hidden layer need not be tuned, which means the input weights and hidden layer neuron threshold are generated randomly before training. Compared with traditional BP and SVM training methods, it is confirmed that ELM provides better generalization at learning speed and human intervene [11]. The output function of ELM with L hidden nodes can be represented as follow:

$$
f(\mathrm{x})=\sum_{i=1}^{L} \beta_{i} G\left(w_{i}, \mathrm{~b}_{i}, \mathrm{x}\right)
$$

Where, $\beta$ denotes the output weight, $w$ denotes the input weight, $g(\mathrm{X})$ denotes the activation function, $\mathrm{b}$ denotes the hidden neuron threshold, $\mathrm{x}$ denotes the input variable. Formula (1) can be simplified as

$$
H \beta=T^{\prime}
$$

where

$$
\begin{gathered}
H\left(\mathrm{w}_{1}, \cdots, \mathrm{w}_{L}, \mathrm{~b}_{1}, \cdots, \mathrm{b}_{L}, \mathrm{x}_{1}, \cdots, \mathrm{x}_{L}\right)= \\
{\left[\begin{array}{c}
g\left(w_{1} \cdot x_{1}+b_{1} \cdots g\left(w_{L} \cdot x_{1}+b_{L}\right)\right. \\
\vdots \\
g\left(w_{1} \cdot x_{N}+b_{N}\right) \cdots g\left(w_{L} \cdot x_{N}+b_{L}\right)
\end{array}\right]_{N \times L}}
\end{gathered}
$$

Given activation function $\mathrm{g}(\mathrm{x})$ satisfying certain mild conditions there exists a sequence of network functions $\{f\}$ approximating to any given continuous target function $\mathrm{f}$ with any expected learning error $\varepsilon>0$, the parameters of w and b are adjusted freely. Parameters $\beta$ can be expressed by

$$
\hat{\beta}=H^{+} T^{\prime}
$$

Where $\mathrm{H}^{+}$is the Moore-Penrose generalized inverse of matrix $\mathrm{H}$.

\section{FEATURES OF PSEUDO INVARIANT LINE MOMENT}

Invariant moments is a method to describe the global shape features, through the method of scale, translation and rotation invariant image feature vector, no need to segment the target, in a certain extent, noise suppression, can effectively reflect the image shape feature.

\subsection{Pseudo Invariant Line Moments}

For digital image $f(\mathrm{x}, \mathrm{y})$, the moments of a region are calculated, which can be proved all of its moments exist. The $\mathrm{p}+\mathrm{q}[12]$ order pseudo line moment is defined follows:

$$
\mu_{p q}=\sum_{i=1}^{N}\left(\mathrm{x}_{i}-\overline{\mathrm{x}}\right)^{\mathrm{p}}\left(\mathrm{y}_{\mathrm{i}}-\overline{\mathrm{y}}\right)^{\mathrm{q}}
$$

Where, $\overline{\mathrm{x}}=\frac{m_{10}}{m_{00}}, \bar{y}=\frac{m_{01}}{m_{00}} 。(\overline{\mathrm{x}}, \overline{\mathrm{y}})$ is $f(\mathrm{x}, \mathrm{y})$ centric position. With zero center distance divided by the high order moment, the normalized center line moment can be defined as follows:

$$
\eta_{p q}=\mu_{p q} / \mu_{00}^{\prime}
$$

The 7 invariant moments can be derived by using 2-order and 3-order normalized center, which are described as follows:

$$
\begin{aligned}
\phi_{1}= & \mu_{20}+\mu_{02} \\
\phi_{2}= & \left(\mu_{20}-\mu_{02}\right)^{2}+4 \mu_{11}^{2} \\
\phi_{3}= & \left(\mu_{30}-3 \mu_{12}\right)^{2}+\left(3 \mu_{21}-\mu_{03}\right)^{2} \\
\phi_{4}= & \left(\mu_{30}+\mu_{12}\right)^{2}+\left(\mu_{21}+\mu_{03}\right)^{2} \\
\phi_{5}= & \left(\mu_{30}-3 \mu_{12}\right)\left(\mu_{30}+\mu_{12}\right)\left[\left(\mu_{30}+\mu_{12}\right)^{2}-\right. \\
& \left.3\left(\mu_{21}+\mu_{03}\right)^{2}\right]+\left(3 \mu_{21}-\mu_{03}\right)\left(\mu_{21}+\right. \\
& \left.\mu_{03}\right)\left[3\left(\mu_{30}+\mu_{12}\right)^{2}-\left(\mu_{21}+\mu_{03}\right)^{2}\right] \\
\phi_{6}= & \left(\mu_{20}-\mu_{02}\right)\left[\left(\mu_{30}+\mu_{12}\right)^{2}-\left(\mu_{21}+\mu_{03}\right)^{2}\right]+ \\
& 4 \mu_{11}\left(\mu_{30}+\mu_{12}\right)\left(\mu_{21}+\mu_{03}\right) \\
\phi_{7}= & \left(3 \mu_{21}-\mu_{03}\right)\left(\mu_{30}+\mu_{12}\right)\left[\left(\mu_{30}+\mu_{12}\right)^{2}-\right. \\
& \left.3\left(\mu_{21}+\mu_{03}\right)^{2}\right]+\left(\mu-3 \mu_{12}\right)\left(\mu_{21}+\right. \\
& \left.\mu_{03}\right)\left[3\left(\mu_{30}+\mu_{12}\right)^{2}-\left(\mu_{21}+\mu_{03}\right)^{2}\right]
\end{aligned}
$$

\subsection{Feature Extraction}

Feature extraction is to will be less susceptible to interference factors of information as the feature of the image calculated. Invariant pseudo line moment integrated boundary feature invariant fast characteristics and regional features of line moment invariant moment characteristics and effective embodiment of the image target characteristic, (6) are obtained for the image of seven feature vector as the target invariant pseudo line moment features. Binary image invariant pseudo line moments feature vector is larger, direct input of classifier will influence the efficiency of model classification. To improve the classifier's classification efficiency, from the feature vector of standard regularization to the interval $[0,1]$.

\section{EXPERIMENTAL RESULTS}

The experimental section aims at evaluating the accuracy and efficiency using the proposed method on the image database of UIUC for car detection. The proposed method is implemented in MATLAB R2008a and the performance of different methods show as table 1 and table 2 [13]. 


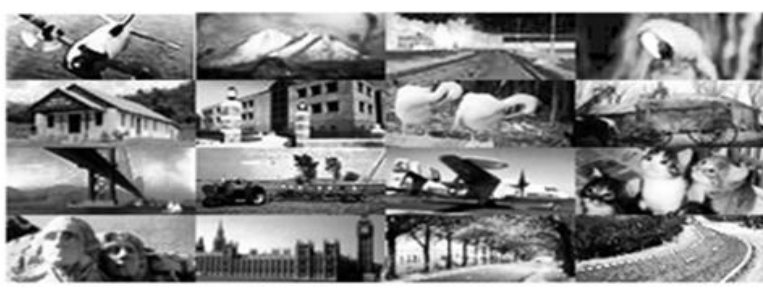

(a) Positive samples

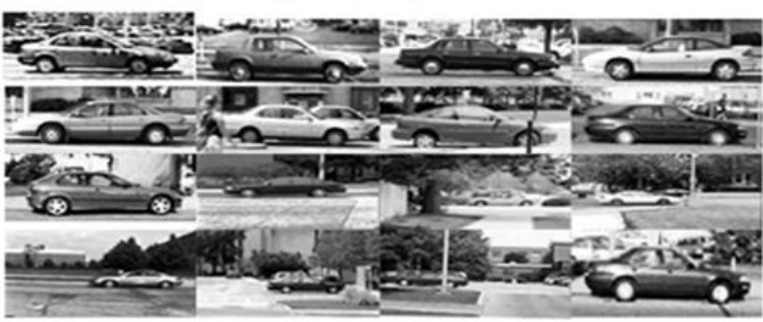

(b) Negative samples

Fig1.samples

Table 1 illustrates the recognition accuracy of different methods including Spare Representation, Boundary Shape Model, CENTRIST, HIK-SVM and PCNN-ELM. The recognition rate of HIK-SVM and PCNN-ELM are reached the $90 \%$ on the single-scale datasets, which can meet the requirements in actual testing. Compared the recognition accuracy, the proposed method achieves better performance. Table 2 illustrates the average time consumed in the classifier test on the datasets. Compared the recognition efficiency, the proposed method consumes less time which means its recognition is faster.

Table 1. The results of different methods

\begin{tabular}{|c|c|c|}
\hline Methods & Single-scale & Multi-scale \\
\hline $\begin{array}{c}\text { Boundary } \\
\text { Shape model }\end{array}$ & $85.0 \%$ & - \\
\hline CENTRIST & $85.0 \%$ & $82.5 \%^{\wedge}$ \\
\hline HIK-SVM & 91.5 & $87.8 \%$ \\
\hline PCNN-ELM & $92.8 \%$ & 89.1 \\
\hline
\end{tabular}

Table2. The average time of test

\begin{tabular}{|c|c|c|}
\hline Methods & Single-scale & Multi-scale \\
\hline Single-scale & $8.3 \mathrm{~s}$ & $2.5 \mathrm{~s}$ \\
\hline Mutli-scale & $174.9 \mathrm{~s}$ & $25.6 \mathrm{~s}$ \\
\hline
\end{tabular}

\section{CONCLUSIONS AND FUTURE WORK}

The vehicle recognition method based on the pseudo line moments and ELM have definite advantages on the accuracy and efficiency from the experiment. The reasons are analysis that the validity of the model is verified through experiments, compared with other algorithms, the recognition accuracy of pseudo invariant linear moment features and ELM vehicle recognition method is higher and the speed is faster. The good performance on accuracy and efficiency of ELM classifier, which provides a new way to identify the vehicle in real-time monitoring system of the vehicle. And the later research direction is based on multi feature fusion of vehicle recognition to improve the accuracy and efficiency of vehicle recognition in complex scenes, which is needed for practical applications exactly.

\section{REFERENCES}

[1] World Health Org., "World Report on Road Traffic Injury Prevention,'[Online] Available:http://www.who.int/violence_injury_preventio n/publications/road_traffic/world_report/factsheets/en/in dex.html

[2] Michalke T P, Gußner T, Bürkle L, et al. Evolution in Advanced Driver Assistance: From Steering Support in Highway Construction Zones to Assistance in Urban Narrow Road Scenarios[M]// Advanced Microsystems for Automotive Applications 2014. Springer International Publishing, 2014:3-13.

[3] Sivaraman S, Trivedi M M. Looking at Vehicles on the Road: A Survey of Vision-Based Vehicle Detection, Tracking, and Behavior Analysis[J]. IEEE Transactions on Intelligent Transportation Systems, 2013, 14(4):17731795.

[4] Cai Y, Hai W. Vision based vehicle detection transfer learning algorithm[J]. Dongnan Daxue Xuebao, 2015, 45(2):275-280.

[5] JIN Li-sheng, WANG Yan, LIU Jing-hua, WANG Ya-li, ZHENG Yi. Front vehicle detection based on Adaboost algorithm in daytime[J]. Journal of Jilin University Engineering and Technology Edition, 2014, 44(6): 16041608

[6] Wang Z, Sun X, Zhang Y, et al. Leaf recognition based on PCNN[J]. Neural Computing \& Applications, 2015:110.

[7] Zhou D, Zhou H, Gao C, et al. Simplified parameters model of PCNN and its application to image segmentation[J]. Formal Pattern Analysis \& Applications, 2015:1-13.

[8] Wang M, Yang L, Wang X, et al. Investigation of Directional Traffic Sign Feature Extracting Based on PCNN in Different Color Space[M]// Multimedia and Signal Processing. Springer Berlin Heidelberg, 2012:2431 .

[9] Nie R, Zhou D, He M, et al. Facial Feature Extraction Using Frequency Map Series in PCNN[J]. Journal of Sensors, 2016, 2016(4):1-9.

[10] Mohammed M M, Badr A, Abdelhalim M B. Image classification and retrieval using optimized PulseCoupled Neural Network[J]. Expert Systems with Applications, 2015, 42(11):4927-4936.

[11] Gao H, Huang G B, Song S, et al. Trends in extreme learning machines: A review[J]. Neural Networks the Official Journal of the International Neural Network Society, 2015, 61:32-48.

[12] Wu J, Geyer C, Rehg J M. Real-time human detection using contour cues[C]// ieee International Conference on Robotics \& Automation. IEEE, 2011:860-867.

[13] Fan X, Piao J, Yu T, et al. Vehicle Detection Method Based on Edge Information and Local Transform Histogram[J]. International Journal of Advancements in Computing Technology, 2013. 